\title{
Route Maintenance Approach for Link Breakage Predicttion in Mobile Ad Hoc Networks
}

\author{
Khalid Zahedi \\ Faculty of Computer Science and Information Systems \\ Universiti Teknologi Malaysia (UTM) \\ Johor, Malaysia
}

\begin{abstract}
Mobile Ad hoc Network (MANET) consists of a group of mobile nodes that can communicate with each other without the need of infrastructure. The movement of nodes in MANET is random; therefore MANETs have a dynamic topology. Because of this dynamic topology, the link breakages in these networks are something common. This problem causes high data loss and delay. In order to decrease these problems, the idea of link breakage prediction has appeared. In link breakage prediction, the availability of a link is evaluated, and a warning is issued if there is a possibility of soon link breakage. In this paper a new approach of link breakage prediction in MANETs is proposed. This approach has been implemented on the well-known Dynamic Source Routing protocol (DSR). This new mechanism was able to decrease the packet loss and delay that occur in the original protocol.
\end{abstract}

Keywords- MANET; link breakage prediction; DSR.

\section{INTRODUCTION}

Mobile Ad hoc Network (MANET) consists of a group of mobile nodes that can be communicated with each other wirelessly without the need to any existed infrastructure. MANETs in general are known with its dynamic topology. The nodes are mobile and their movement is random. MANET's dynamic topology makes link breakages a frequent habit. This habit causes many problems such as data loss, delay, and others which degrade the performance of the MANETs protocols. In order to reduce the damage size of this phenomenon, the idea of link breakage prediction has appeared.

In link breakage prediction, a link breakage can be predicted before its real occurring so route maintenance can start before the occurring of the problem avoiding the problems that come with a link breakage. In the link breakage prediction, a node in an active route can predict if the link between it and its previous hop will break soon. In this case it can inform the source node about the problem and the source node, if still needs the route, will be able to construct a new route which avoids this soon to be broken link. It has been found that this procedure has made a good improvement in the performance of the mobile ad hoc network's protocols, but the problem is that the focusing during constructing a new route was only on excluding the link that was predicted to have a link breakage. This mechanism may cause constructing a new

\author{
Abdul Samad Ismail \\ Faculty of Computer Science and Information Systems \\ Universiti Teknologi Malaysia (UTM) \\ Johor, Malaysia
}

route with some or all bad links from the current used route which are weak but did not predicted to be broken yet. These links may break during or directly after the constructing of the new route which will cause a high decrease in the packet delivery ratio and a high increase in the packet loss and delay. In order to improve the idea of link breakage prediction, this paper has proposed a new approach for link breakage prediction in MANETs. In this new approach, the source node of an active route, after being informed about a link breakage in its current used route, will construct a new route which avoids the use of any link from the current used route. That means excluding all the links in the current route, or in other words, excluding the whole current used route not just the soon to be broken link. So, the new constructed route will be completely different from the current used one. This approach is novel and it has been implemented on the well-known reactive routing protocol Dynamic Source routing Protocol (DSR).

This paper is organized in seven sections: Section I is an introduction. Section II gives some examples of the works that have been done in this area. Section III gives a description about the Dynamic Source Routing protocol (DSR). Section IV illustrates the proposed idea. Section V discusses the simulation environment. Section VI detailed the results that have obtained. Section VII concludes this paper, and section VIII provides some future works.

\section{LITERATURE REVIEW}

Several researchers have investigated the area of link breakage prediction in mobile ad hoc networks. In this section, some examples of their works are discussed.

Ramesh et al. [1] have studied the problem of link breakage prediction in the DSR routing protocol. Their idea is that during the route discovery process, the source node builds two routes which are the source route and another route can be used as a backup. The backup route can be used if the primary route (source route) was predicted to have a link breakage soon.

Li et al. [2] have studied the link prediction in the AODV routing protocol by establishing a signal intensity threshold which is Pr-THRESHOLD. If the received signal intensity is lower than the threshold, the upstream node will calculate the distance between it and the sending node through the intensity of the received packet signal, and estimate the relative velocity between it and the sending node through the time difference of 
the neighboring received data and the intensity of the packet signal. Then, according to the relative position and the relative velocity with the sending node, a node can estimate when to send a RRER to the sending node to warning it about a link failure. When the source node received this RRER message, it will start its restored process searching its routing table and find another route to the destination.

Qin \& Kunz [3] have dealt with the problem of link failure prediction by proposing an equation to calculate the exact time that a link breakage can occur. They named their method the link breakage prediction algorithm. In their idea, each node maintains a table that contains the previous hop node address, the value of the received packet signal power, and the time which this data packet has been received. After receiving three data packets, a node will calculate the link breakage time and compare it with a fixed threshold. If the node predicted that the link with its previous neighbor will have a link breakage soon, it will send a warning message to the source node of the active route to warn it about the link breakage probability. If the source still needs the route it will perform a route discovery process to establish a new route to the destination. Their idea has been implemented using DSR routing protocol.

Zhu [4] has studied the problem of link breakage prediction by using the same equation that have been proposed by Qin \& Kunz [3] which is the link breakage prediction algorithm, but she has implemented this algorithm using the AODV and MAODV routing protocols

Choi et al. [5] has dealt with the problem of link breakage prediction in vehicular ad hoc network. They proposed an algorithm to predict a link breakage possibility using the value of the RSSI (Received Signal Strength Indicator). Each vehicle in the network periodically scans the received signals from its neighbors and uses the collected value to calculate the distance, the velocity, and the acceleration of its next hop which it receives data packets from. By calculating these three values, the node can predict if a link breakage will occur, and can determine if the effected link can be maintained or a new link is needed to be constructed. If the effected vehicle found that a link breakage in the link with its next hop will occur, it will use one of its neighbors which has the highest value of RSSI with (that means the one which is the nearest to it) to build a new link with before the previous link with its other neighbor becomes broken.

Goff et al. [6] have studied the link breakage problem in the DSR routing protocol. They defined a region they named it the preemptive region, and they also defined a threshold which they named it the preemptive threshold, they defined this threshold as the signal power of the received packets at the edge of the preemptive region. When a node enters the preemptive region it will send a warning message to the source node of the active route in order to inform it that a link breakage will soon occur. So if the source is still interesting with the route, it will generate a route discovery process to establish a new route without that soon to be broken link.

Ouni et al. [7] studied the problem of link breakage prediction in the DSR routing protocol and tried to propose a solution by proposing a check model composed of two modules. The first module includes performing different simulations to have an idea about the nodes behavior and by this allowing determining the suitable routes to use, while the second model checks the path availability and the deadline delay satisfaction. This check model was also used to predict the validity periods of the selected path and the satisfaction of the delay constrains.

Lu et al. [8] have worked on the DSR routing protocol and proposed a mechanism for switching to a new route if the current route is found to have a link breakage soon. Their mechanism which is named DSR-link switch (DSR-LS) first detects a link breakage between a nod and its next hop to the source by measuring the power of the received packets. If a link failure is detected to occur soon, the node, using this mechanism, will send a link switch request (LSRE) in one hop range to search appropriate nodes that act as relaying stations or bridge nodes. This LSRE request will be sent by including it in the RTS/CTS packets of the MAC layer during the current communication. After finding a new strong links, the current route will be shift to a more stable path.

\section{DYNAMIC SOURCE ROUTING (DSR)}

The Dynamic Source Routing (DSR) is a simple and efficient routing protocol designed to be used specifically in mobile ad hoc networks. Through using DSR, the network is completely self-organizing and self-configuring. Network nodes cooperate to forward packets for each other to allow communication over multiple hops between the nodes that are not located within the transmission range of each other. As nodes in the mobile ad hoc network move about, join or leave the network, and as wireless transmission conditions such as types of interference change, all routing is automatically determined and maintained by the DSR routing protocol.

The DSR routing protocol applies the idea of source routing, this idea can be summarized by sending the whole route from the source node to the destination node in each transmitted IP packet, so the intermediate nodes will have to only forward these packets without taking any routing decision. In order to implement the idea of source routing, DSR makes use of special header for carrying control information which can be included in any IP packet. This header is named DSR options header [9].

The DSR options header is a header existed in any sent IP packet by a node implements DSR routing algorithm. This header must immediately follow the IP header in the sent packet. It consists of two fields, fixed length field and variable length field. The fixed length field is a 4-octet portion that has four fields (Next Header, F, Reserved, Payload Length) while the variable length field is called the options field, which has zero or more pieces of optional information which are called DSR options. In DSR routing protocol there are eight types of options, each one of them must be included in a DSR options header in order to be transmitted along the network.

DSR options header is located in an IP packet directly after the IP header and before any other header in the packet. It can contain one or more of the following options: 
1) Route Request option.

2) Route Reply option.

3) Route Error option.

4) Acknowledgement request option.

5) Acknowledgement option.

6) DSR source route option.

7) Padl option.

8) PadN option.

The DSR protocol composes of two basic mechanisms which work together to allow the discovery and maintenance of the source routes in mobile ad hoc networks. These two basic mechanisms are:

\section{1) Route discovery}

\section{2) Route maintenance}

Route discovery is the mechanism that is used by a source node which wishes to send data packets to a destination node which has no route to it in its route cache. Using this mechanism the source node can obtain a source route to the destination.

Route maintenance is the mechanism that is used by a source node to detect a link breakage along its source route to a destination node. Using this mechanism the source node can know if it can still use the route or not. When the source node indicates the existence of a broken link in the source route, it can use another route or trigger a new route discovery process. Route maintenance is used only with active routes.

Route discovery and route maintenance mechanisms each operates entirely on demand. Unlike other protocols, DSR does not require periodic packets of any kind at any level within the network. For example, DSR does not use any periodic routing advertisement and does not use neighbor detection messages. This is a full on demand behavior.

It is possible that a link may not work equally well in both directions because of antenna, or propagation patterns, or sources interference. These types of links are called unidirectional links. The routes that compose of such type of links are called asymmetric routes or paths. DSR allows unidirectional links to be used when necessary; this improves the overall performance and the network connectivity.

DSR also supports the internetworking between different types of wireless networks allowing a source route to be composed of hops over a combination of any types of networks available [10]. As an example, some nodes in the ad hoc network may have only short-range radios, while other nodes have both short-range and long-range radios; the combination of these nodes together can be considered by DSR as a single ad hoc network.

\section{THE PRoposed IdEA}

In this section a new approach for the link breakage prediction in the mobile ad hoc networks will be introduced. The idea is to construct a new route which is completely different from the current used route by excluding all the links exist in the current used one. So during the phase of constructing the new route if another link or other links have been predicted to be broken, there will be no need for trying to avoid this link or these links, because from the beginning, the new constructed route has excluded all the links in the previous route. The approach's idea is as follows:

Each node along an active source route scans the received data packets signals from its previous hop node. When a node found that the Received Signal Strength Indicator (RSSI) value of the received data packets from its previous hop is still decreasing after three successive measurements, the node will realize that the link between it and its previous hop will have a link breakage soon. In this case it will generate a packet and initialize a new option which will be named Soon Link Breakage warning (SLBW). This option will be inserted in the options field of the DSR options header of the packet. Then, this packet which can be named SLBW message will be unicasted to the source node of this active route to indicate to it that a link breakage along this route will occur. The SLBW option is similar to the RERR option of the DSR routing protocol with some modifications, the error type in the SLBW will be set to (4) in order to indicate the link breakage probability. SLBW will include the source node's address in order to reach the source of the affected route in case more than one route share some of the links of the affected route, and will also include the addresses of both, the node that predicted the link breakage and its previous hop node's address. By sending the addresses of the nodes at the end of the soon to be broken link, the source node will be able to determine which route will have a link breakage. When the source node receives the SLBW message, if it still needs the route, it will set the route that has a soon to be broken link with the state of Route with a Breakage Prediction (RBP) in its route cache. Then it will check its route cache to see if it has another route to the destination. If it has one, it will make a match between the intermediate node addresses of the cached route and the node addresses in the current used route which has the state (RBP). If there was no match, the source starts sending data packets using this new source route. Otherwise, it will trigger a route discovery process by broadcasting to its neighbors a Modified Route Request (MRREQ) message. The MRREQ message is an IP packet generated by the source node which its DSR options header contains two options, the RREQ option and the source route option.

In the source route option, the source node will append the route with the (RBP) state. This step is made by the source node in order to discover a new route which has no any relationship with the current used route which has the state (RBP), because the current route may have other weak links. Each node receives this MRREQ message will check first if it is the destination of this MRREQ. If it is the destination, it will initialize a RREP option similar to the one in the original DSR routing protocol. Else, it will check if it has received this message before, so if the RREQ option in the received MRREQ message has the same source address and REQUEST ID of a previous received one, or if the receiving node found its address appended in the RECORD of the received option, it will discard this message. Otherwise, the node will check if its address is appended in the source route option of the MRREQ message. If it found its address appended, it will discard the MRREQ message. Else, it will append its address 
in the RECORD of the RREQ option in the MRREQ message and rebroadcast the message to its neighbors.

In Fig. 1, in order to construct a route which has no any relationship with the current used one, when node 1 receives the MRREQ message it will make a match between its address and the addresses in the source route option of the MRREQ message. So when it found its address appended, it will discard the message and not forward it any more. The same situation will repeat with the other nodes of the route.

\section{Simulation}

In this section the parameters that have been manipulated, the metrics that have been used for comparison, and the environment that has been used to make the experiments will be discussed in detail.

\section{THE USED PARAMETERS}

From our literature review, we found that most of the other papers have used three parameters for making their comparisons; these parameters are (number of nodes in the network, simulation time, and pause time). In order to make new and unique comparisons, we used in this paper three other parameters which we found that no other paper in the link prediction area has used before. These three parameters are:

1) Number of nodes per route.

2) Node mobility speed.

3) Node transmission range.

\section{THE Used Metrics}

In this paper three metrics have been used in order to make the comparisons between the two protocols. These metrics are:

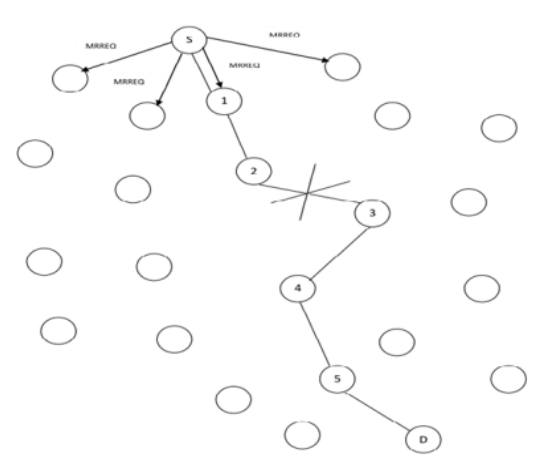

Figure1. A clarification to the idea

1) Packet Delivery Ratio.

2) Number of dropped data packets.

3) Average End to End Delay.

The following is the definision of each metric:

- Packet Delivery Ratio: It is the ratio between the number of received data packets by the destination and the number of generated data packets by the source.
- Number of dropped data packets: It is the number of data packets that have failed to arrive successfully to the destination.

- Average End to End Delay: It is the time that is taken by a packet in order to transfer from a source node to a destination node.

\section{THE USED ENVIRONMENT}

As we mentioned, the simulations in this paper have been carried out by varying three parameters. When any parameter (of the three used parameters) is manipulated, all the other parameters will be fixed. See Table I.

TABLE I. SIMULATION PARAMETERS

\begin{tabular}{|l|c|}
\hline Parameter & Value \\
\hline Number of nodes in the network & 1000 \\
\hline Number of nodes per route & $10-100$ \\
\hline Mobility speed & $10-100 \mathrm{~m} / \mathrm{s}$ \\
\hline Transmission range & $750-10 \mathrm{~m}$ \\
\hline Simulation time & $80 \mathrm{sec}$ \\
\hline Pause time & $5 \mathrm{~ms}$ \\
\hline Terrain size & $1000 \times 1000$ \\
\hline Traffic mode & CBR \\
\hline Packet size & 1300 byte \\
\hline Packet sending rate & $5 \mathrm{p} / \mathrm{s}$ \\
\hline MAC protocol & IEEE 802.11 \\
\hline Mobility model & Random Waypoint \\
\hline Antenna type & Omni-directional \\
\hline Simulator & NCTUns 6.0 \\
\hline
\end{tabular}

VI. RESULTS AND DISCUSSION

In this section, the achieved results will be discussed in detail. In figure 2, we can see that the (Packet delivery ratio) is decreasing for both protocols as the number of nodes per route is increasing, but the decreasing in the case of (DSR modified) is much less than the decreasing in the (DSR original). The reason of decreasing in the PDR is that when the number of nodes in the route increases this means that the number of links in that route also increases, so the probability of link breakages occurrence also increases. Also, we can notice that the difference in PDR between the two protocols is big when the number of nodes per route is low (as it is clear when there is 10 nodes), but this difference is reduced gradually as the number of nodes per route increases (as it is clear when there is 100 nodes). The reason behind this is that the increase in the number of nodes per route reduces the efficiency of the new mechanism where link breakages will so frequently occur.

In figure 3 , we can see that the (Number of dropped data packets) is increasing for both protocols as the number of nodes per route is increasing, but the increasing in the case of (DSR modified) is much less than the increasing in the (DSR original). The reason of increasing in the number of dropped data packets is that when the number of nodes in the route increases this means that the number of links in that route also increases, so the probability of link breakages occurrence also increases. Also, we can notice that the difference in the number of dropped data packets between the two protocols is 
big when the number of nodes per route is low (as it is clear when there is 10 nodes), but this difference is reduced gradually as the number of nodes per route increases (as it is clear when there is 100 nodes). The reason behind this is that the increase in the number of nodes per route reduces the efficiency of the new mechanism where link breakages will so frequently occur.

In figure 4, we can see that the (Average End to End Delay) is increasing for both protocols as the number of nodes per route is increasing, but the increasing in the case of (DSR modified) is much less than the increasing in the (DSR original). The reason of increasing in the average end to end delay is that when the number of nodes in the route increases this means that the number of links in that route also increases, so the probability of link breakages occurrence also increases. Also, we can notice that the difference in the average end to end delay between the two protocols is big when the number of nodes per route is low (as it is clear when there is 10 nodes), but this difference is reduced gradually as the number of nodes per route increases (as it is clear when there is 100 nodes). The reason behind this is that the increase in the number of nodes per route reduces the efficiency of the new mechanism where link breakages will so frequently occur.

In figure 5, we can see that the (Packet delivery ratio) is decreasing for both protocols as the mobility speed of nodes is increasing, but the decreasing in the case of (DSR modified) is much less than the decreasing in the (DSR original). The reason of decreasing in the PDR is that the increase in the mobility speed of nodes forming a route means the increase in the link breakages in the links between those nodes. Also, we can notice that the difference in PDR between the two protocols is big when the mobility speed of nodes is low (as it is clear when it is $10 \mathrm{~m} / \mathrm{s}$ ), but this difference is reduced gradually as the mobility speed increases (as it is clear when it is $100 \mathrm{~m} / \mathrm{s}$ ).

In figure 6, we can see that the (Number of dropped data packets) is increasing for both protocols as the mobility speed of nodes is increasing, but the increasing in the case of (DSR modified) is much less than the increasing in the (DSR original). The reason of increasing in the number of dropped data packets is that the increase in the mobility speed of nodes forming a route means the increase in the link breakages in the links between those nodes. Also, we can notice that the difference in the number of dropped data packets between the two protocols is big when the mobility speed of nodes is low (as it is clear when it is $10 \mathrm{~m} / \mathrm{s}$ ), but this difference is reduced gradually as the mobility speed increases (as it is clear when it is $100 \mathrm{~m} / \mathrm{s}$ ). The reason behind this is that the increase in the mobility speed of nodes of the route reduces the efficiency of the new mechanism where link breakages will so frequently occur.

In figure 7, we can see that the (Average End to End Delay) is increasing for both protocols as the mobility speed of nodes is increasing, but the increasing in the case of (DSR modified) is much less than the increasing in the (DSR original). The reason of increasing in the average end to end delay is that the increase in the mobility speed of nodes forming a route means the increase in the link breakages in the links between those nodes. Also, we can notice that the difference in the average end to end delay between the two protocols is big when the mobility speed of nodes is low (as it is clear when it is $10 \mathrm{~m} / \mathrm{s}$ ), but this difference is reduced gradually as the mobility speed increases (as it is clear when it is $100 \mathrm{~m} / \mathrm{s}$ ). The reason behind this is that the increase in the mobility speed of nodes of the route reduces the efficiency of the new mechanism where link breakages will so frequently occur.

In figure 8 , we can see that the (Packet delivery ratio) is decreasing for both protocols as the transmission range of nodes is decreasing, but the decreasing in the case of (DSR modified) is much less than the decreasing in the (DSR original). The reason behind the decreasing in the PDR is that the decrease in the transmission range of nodes in a route means that the links between those nodes will be weaker, so the probability of breaking such links will be higher. Also, we can notice that the difference in PDR between the two protocols is big when the transmission range of nodes is high (as it is clear when it is $750 \mathrm{~m}$ ), but this difference is reduced gradually as the transmission range decreases (as it is clear when it is $10 \mathrm{~m}$ ). The reason behind this is that the decrease in the transmission range of nodes of the route reduces the efficiency of the new mechanism where link breakages will so frequently occur.

In figure 9, we can see that the (Number of dropped data packets) is increasing for both protocols as the transmission range of nodes is decreasing, but the increasing in the case of (DSR modified) is much less than the increasing in the (DSR original). The reason behind the increasing in the number of dropped data packets is that the decrease in the transmission range of nodes in a route means that the links between those nodes will be weaker, so the probability of breaking such links will be higher. Also, we can notice that the difference in the number of dropped data packets between the two protocols is big when the transmission range of nodes is high (as it is clear when it is $750 \mathrm{~m}$ ), but this difference is reduced gradually as the transmission range decreases (as it is clear when it is 10 $\mathrm{m})$. The reason behind this is that the decrease in the transmission range of nodes of the route reduces the efficiency of the new mechanism where link breakages will so frequently occur.

In figure 10, we can see that the (Average End to End Delay) is increasing for both protocols as the transmission range of nodes is decreasing, but the increasing in the case of (DSR modified) is much less than the increasing in the (DSR original). The reason behind the increasing in the average end to end delay is that the decrease in the transmission range of nodes in a route means that the links between those nodes will be weaker, so the probability of breaking such links will be higher. Also, we can notice that the difference in the average end to end delay between the two protocols is big when the transmission range of nodes is high (as it is clear when it is $750 \mathrm{~m}$ ), but this difference is reduced gradually as the transmission range decreases (as it is clear when it is $10 \mathrm{~m}$ ). The reason behind this is that the decrease in the transmission range of nodes of the route reduces the efficiency of the new mechanism where link breakages will so frequently occur. 




Figure 2 PDR and No. of nodes per route

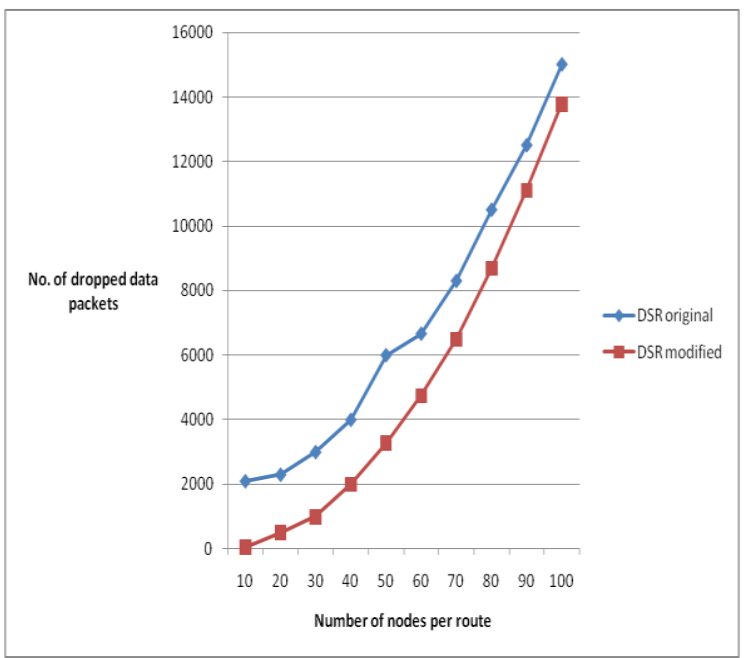

Figure 3 No. of dropped packets and No. of nodes per route

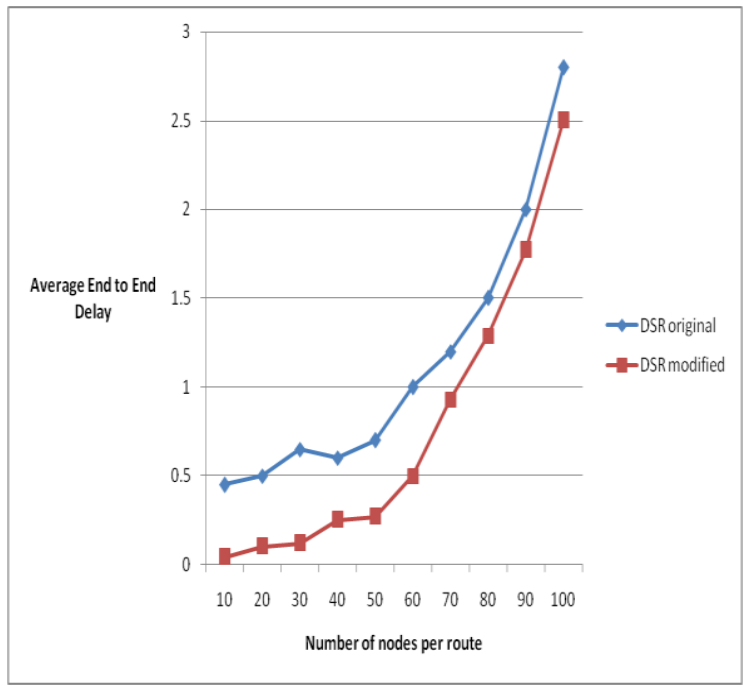

Figure 4 Delay and No. of nodes per route

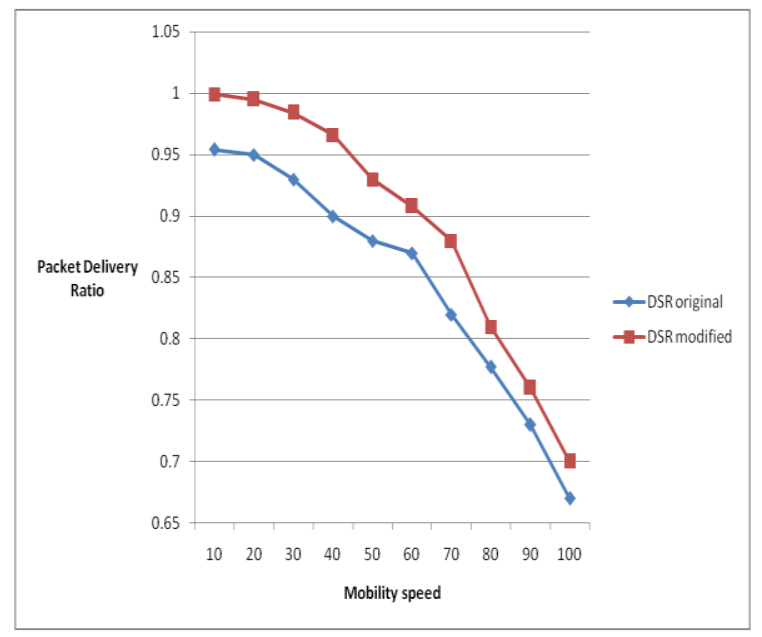

Figure 5 PDR and mobility speed

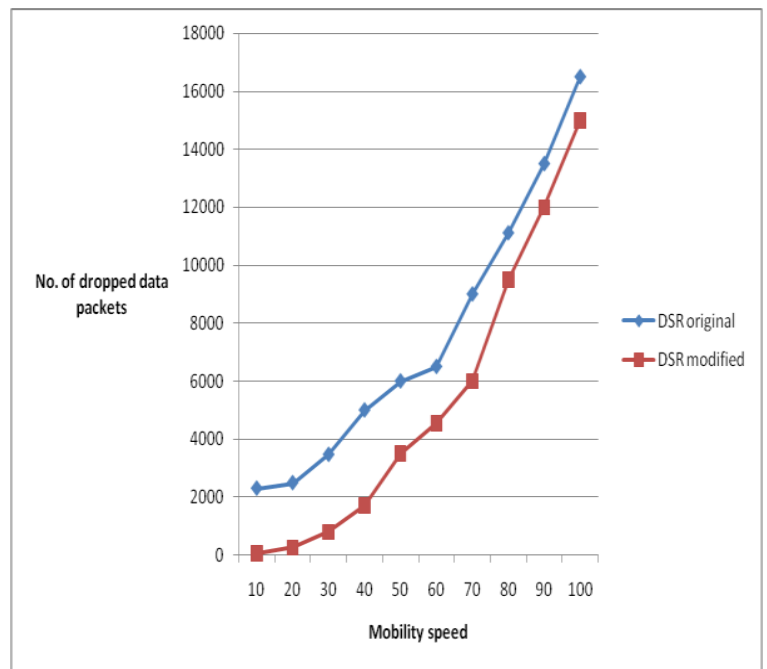

Figure 6 No. of dropped packets and mobility speed

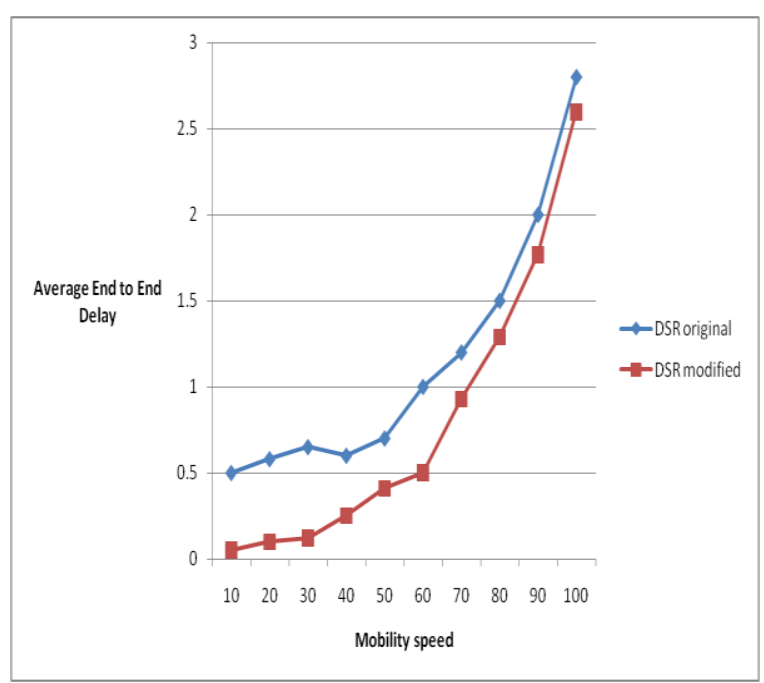

Figure 7 Delay and mobility speed 

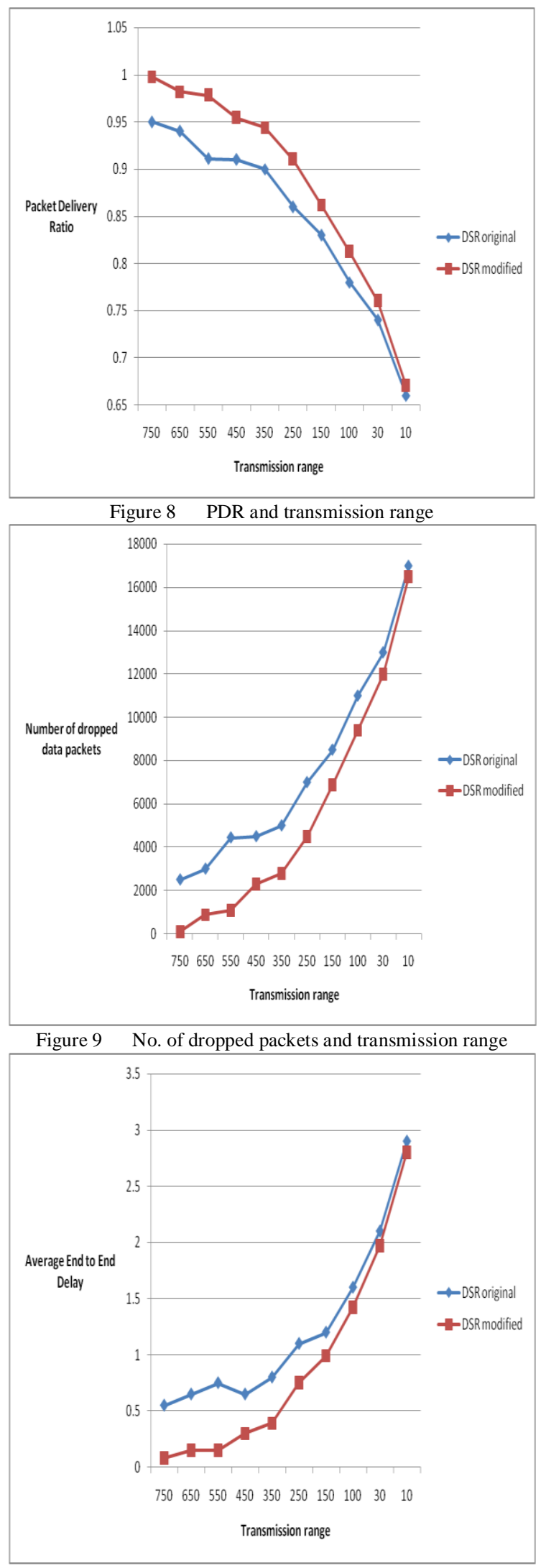

Figure 10 Delay and transmission range

\section{CONCLUSION}

Many approaches have been proposed to deal with the idea of link breakage prediction, but the problem is that all the previous approaches were building a new route that avoids using only the same soon to be broken link, but no one of these approaches was able to build a new route which avoids all the other links in the old route. In this paper, a new approach for solving the problem of link breakages in MANET has been proposed and implemented on the Dynamic Source Routing (DSR) routing protocol. In this approach, the Received Signal Strength Indicator (RSSI) value will be used by a node along an active route to predict a link breakage in its link with its next hop to the source node of this active route. The node will warn the source node, and the source (if it still needs the route) will discover a new route without using any link from the current route which has a soon to be broken link. The idea behind this is to reduce the probability of constructing a route with bad links which can break during or directly after the constructing of a new route. It has been found that this approach was able to increase the packet delivery ratio and decrease both the packet loss and the end to end delay comparing to the DSR routing protocol. So, this approach was able to improve the performance of the protocol.

\section{FUTURE WORK}

As a future work, this work can be extended by using other metrics for making the comparisons between the original and modified DSR routing protocols such as the terrain size, packet size, packet sending rate, and others. Also, the traffic mode can be changed from CBR to VBR and find the difference. Another change can be made to the mobility model. In this work the mobility model that has been used is the random way point mobility model, so another research can be done by using other mobility models such as the random walk mobility model, or the random direction mobility model, and see the difference.

\section{REFERENCES}

[1] Ramesh, V., Subbaiah, P., and Supriya, K. (2010). Modified DSR (preemptive) to reduce link breakage and routing overhead for MANET using proactive route maintenance (PRM). Global Journal of Computer Science and Technology. Vol. 9. Issue 5, 124-129.

[2] Li, Q., Liu, c., and Jiang, H. (2008). The routing protocol AODV based on link failure prediction. ICSP IEEE. 2008.

[3] Qin, L., and Kunz, T. (2002). Increasing packet delivery ratio in DSR by link prediction. HICSS 03. IEEE. 2002. Hawaii.

[4] Zhu, Y. (2002). Proactive connection maintenance in AODV and MAODV. Master of Science. Carleton University, Canada.

[5] Hoi, W., Nam, J., and Choi, S. (2008). Hop state prediction method using distance differential of RSSI on VANET. NCM 2008. IEEE. 426431.

[6] Goff, T., Abu-Ghazaleh, N., Phatak, D., and Kahvecioglu, R. (2003). Preemptive routing in ad hoc networks. Journal of Parallel and Distributed Computing. 63 (2003), 123-140.

[7] Ouni, S., Bokri, J., and Kamoun, F. (2009). DSR based routing algorithm with delay guarantee for ad hoc networks. Journal of Networks. Vol. 4(5), 359-369.

[8] Lu, H., Zhang, J., and Luo, X. (2008). Link switch mechanism based on DSR route protocol. ICINIS IEEE. 2008.

[9] Internet Engineering Task Force (IETF), Dynamic Source Routing (DSR) protocol, 2007. http://www.ietf.org 
[10] Sarkar, S. K., Basavaraju, T., Puttamadappa, C. (2008). Ad hoc mobile wireless networks. (First ed.). New York: Auerbach Publications.

[11] Rajabhushanam, C., \& Kathirvel, A. (2011). Survey of Wireless MANET Application in Battlefield Operations. IJACSA - International Journal of Advanced Computer Science and Applications, 2(1).

[12] Multicasting over Overlay Networks - A Critical Review. IJACSA International Journal of Advanced Computer Science and Applications, 2(3), 54-61.
AUTHORS PROFILE

Khalid Zahedi is a Master's student in the faculty of Computer Science and Information Systems in UTM. His research interests include: Mobile Ad hoc Networks (MANETs) and Vehicular Ad hoc Networks (VANETs).

Abdul Samad Ismail is an associate professor in the faculty of Computer Science and Information Systems in UTM. His research interests include: Mobile Ad hoc Networks (MANETs), Vehicular Ad hoc Networks (VANETs), Wireless Sensor Networks (WSNs), and Network Security. 